\title{
A constraint-separation principle in model predictive control ${ }^{\star}$
}

\author{
Uroš V. Kalabića ${ }^{a}$ Ilya V. Kolmanovsky ${ }^{\mathrm{b}}$ \\ ${ }^{\mathrm{a}}$ Mitsubishi Electric Research Laboratories, 201 Broadway, Cambridge, MA 02139 \\ ${ }^{\mathrm{b}}$ Department of Aerospace Engineering, University of Michigan, 1320 Beal Avenue, Ann Arbor, MI 48109
}

\begin{abstract}
In this brief, we consider the constrained optimization problem underpinning model predictive control (MPC). We show that this problem can be decomposed into an unconstrained optimization problem with the same cost function as the original problem and a constrained optimization problem with a modified cost function and dynamics that have been precompensated according to the solution of the unconstrained problem. In the case of linear systems subject to a quadratic cost, the unconstrained problem has the familiar LQR solution and the constrained problem reduces to a minimum-norm projection. This implies that solving linear MPC problems is equivalent to precompensating a system using LQR and applying MPC to penalize only the control input. We propose to call this a constraint-separation principle and discuss the utility of both constraint separation and general decomposition in the design of MPC schemes and the development of numerical solvers for MPC problems.
\end{abstract}

Key words: Constrained systems; model predictive control; constrained control system design.

\section{Introduction}

Model predictive control (MPC) is an optimization-based framework for determining constraint-admissible, stabilizing control inputs to open-loop control systems (Rawlings et al., 2017). Conventionally, MPC is applied according to the schematic in Fig. 1 and presented as a constraint-enforcing, feedback control scheme, which can simultaneously stabilize a system and enforce constraints on that system. This is in contrast to other constraint-enforcing schemes, such as reference governors (Garone et al., 2017), which are only used to enforce constraints in precompensated systems, and anti-windup schemes (Kothare et al., 1994) and barrier-function methods (Tee et al., 2009), which are used to modify stabilizing control designs in order to enforce constraints.

In this paper, we propound the perspective that MPC is not substantially different from the alternative schemes. This is because the optimization problem solved by MPC can be decomposed into two separate optimization problems, the solution to one ensuring stability of the inner-loop, and the solution to the other computing an outer-loop modification that enforces constraints. In the case of linear systems subject to a quadratic penalty, the inner-loop compensation has the familiar closed-form solution of the discrete-time linear-quadratic regulator (LQR) (Dorato and Levis, 1971) and the constraint-enforcing, outer-loop problem is reduced to a minimum-norm projection, without a terminal cost term.

The above implies that linear MPC can be interpreted as an add-on, constraint enforcing mechanism similar to the extended command governor (ECG) (Gilbert and Ong, 2011). This was first shown by Kouvaritakis et al. (2000) for the time-invariant case, i.e., where the dynamics are time-invariant and the terminal cost is obtained as the solution

\footnotetext{
‡ This paper was not presented at any IFAC meeting. Corresponding author U. V. Kalabić.

Email addresses: kalabic@umich.edu (Uroš V. Kalabić), ilya@umich.edu (Ilya V. Kolmanovsky).
} 


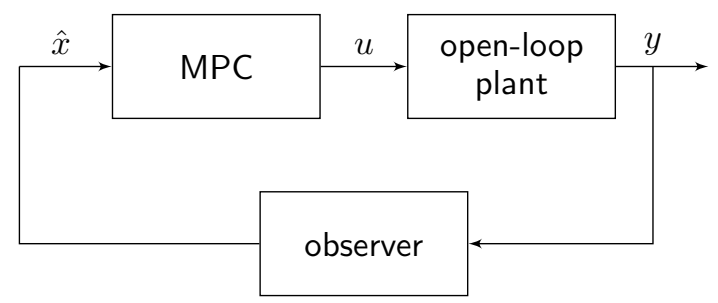

Fig. 1. MPC applied to an open-loop plant

to the discrete-time, infinite-horizon LQR problem, and the benefits of this approach for numerical implementation were discussed by Kouvaritakis et al. (2002); Rossiter (2004); Rossiter et al. (2010). Here we show the same result in the case of linear time-varying systems, which is important because numerical strategies for nonlinear MPC problems often involve sequentially solving linear time-varying MPC problems. We refer to the result as a constraint-separation principle and discuss its implications.

In addition to the above result, we consider decomposition in the general nonlinear setting. We show that constraint separation is not generally possible in the case of nonlinear MPC problems, since the initial MPC problem is decomposed into an unconstrained problem requiring a convenient closed-form solution, which does not generally exist, and another MPC problem that does not necessarily have a convenient structure. Nevertheless, we show that if the MPC problem has a locally linear-quadratic structure, then constraint separation holds locally.

The paper is structured as follows. Section 2 derives the decomposition of nonlinear MPC into stabilizing and constraint-enforcing optimization problems. Section 3 derives the constraint-separation principle for locally linear MPC problems. Section 4 discusses the implications of the constraint-separation principle. Section 5 is the conclusion.

\section{MPC optimization problem}

The optimization problem we consider is given by Rawlings et al. (2017),

$$
\begin{aligned}
\min _{u} & V_{f}\left(x_{N}\right)+\sum_{k=0}^{N-1} L_{k}\left(x_{k}, u_{k}\right), \\
\text { sub. to } & x_{k+1}=f_{k}\left(x_{k}, u_{k}\right), \\
& \left(x_{k}, u_{k}\right) \in \mathcal{C}_{k}, \forall k \in \mathbb{Z}_{N}, \\
& x_{N} \in \mathcal{X}_{N}
\end{aligned}
$$

where $x_{0}$ is given, $f_{k}: \mathbb{R}^{n} \times \mathbb{R}^{m} \rightarrow \mathbb{R}^{n}$ is continuous, $f_{k}(0,0)=0$, and $\mathcal{C}_{k}$ is closed for all $k \in \mathbb{Z}_{N}$ where $\mathbb{Z}_{N}$ is the set of the first $N$ non-negative integers. The cost functions $V_{f}: \mathbb{R}^{n} \rightarrow \mathbb{R}$ and $L_{k}: \mathbb{R}^{n} \times \mathbb{R}^{m} \rightarrow \mathbb{R}$ are continuous and locally bounded, and satisfy the following properties,

$$
\begin{aligned}
& L_{k}(0,0)=V_{f}(0)=0, \\
& L_{k}(x, u) \geq \alpha(\|u\|), V_{f}(x) \geq 0,
\end{aligned}
$$

for all $x \in \mathbb{R}^{n}, u \in \mathbb{R}^{m}, k \in \mathbb{Z}_{N}$, where $\alpha$ is a $\mathcal{K}_{\infty}$-function (Rawlings et al., 2017). The assumptions are required to ensure the existence of a solution to the optimization problem. We note that our subsequent results do not dependent on other, standard assumptions found in the MPC literature (Rawlings et al., 2017), like those which ensure that the solution is recursively feasible and results in a stabilizing control law. We also note that we make no additional assumptions on the geometry of the sets $\mathcal{C}_{k}$ such as, for example, convexity.

We define the sets,

$$
\begin{aligned}
& \overline{\mathcal{C}}_{k}:=\left\{(x, u):(x, u) \in \mathcal{C}_{k}, f_{k}(x, u) \in \mathcal{X}_{k+1}\right\}, \\
& \overline{\mathcal{C}}_{k}(x):=\left\{u:(x, u) \in \overline{\mathcal{C}}_{k}\right\}
\end{aligned}
$$

for all $k \in \mathbb{Z}_{N}$, where $\mathcal{X}_{k}:=\operatorname{Proj}_{\mathbb{R}^{n}} \mathcal{C}_{k}$. These sets are closed for all $x \in \mathbb{R}^{n}$ due to the closedness of $\mathcal{C}_{k}$ and $\mathcal{X}_{k+1}$ and the continuity of $f_{k}$. 
A sequence of control inputs $u^{*}$ solving (1) satisfies (Rawlings et al., 2017),

$$
u_{k}^{*} \in \underset{u \in \overline{\mathcal{C}}_{k}\left(x_{k}^{*}\right)}{\arg \min } V_{k+1}\left(f_{k}\left(x_{k}^{*}, u\right)\right)+L_{k}\left(x_{k}^{*}, u\right),
$$

for all $k \in \mathbb{Z}_{N}$, with $x_{k+1}^{*}=f_{k}\left(x_{k}^{*}, u_{k}^{*}\right)$ and $x_{0}^{*}=x_{0}$, where $V_{k}$ satisfies the Bellman equation,

$$
V_{k}(x)=\min _{u \in \overline{\mathcal{C}}_{k}(x)} V_{k+1}\left(f_{k}(x, u)\right)+L_{k}(x, u)
$$

with domain $\overline{\mathcal{X}}_{k}:=\operatorname{Proj}_{\mathbb{R}^{n}} \overline{\mathcal{C}}_{k}$, and $V_{N}=\left.V_{f}\right|_{\mathcal{X}_{N}}$.

Consider the optimization problem (1) without inequality constraints (1c)-(1d). A sequence of control inputs $\tilde{u}^{*}$ solving this problem satisfies,

$$
\tilde{u}_{k}^{*} \in \underset{u}{\arg \min } \tilde{V}_{k+1}\left(f_{k}\left(\tilde{x}_{k}^{*}, u\right)\right)+L_{k}\left(\tilde{x}_{k}^{*}, u\right)
$$

for all $k \in \mathbb{Z}_{N}$, with $\tilde{x}_{k+1}^{*}=f_{k}\left(\tilde{x}_{k}^{*}, \tilde{u}_{k}^{*}\right)$ and $\tilde{x}_{0}^{*}=x_{0}$, where $\tilde{V}_{k}$ satisfies the Bellman equation,

$$
\tilde{V}_{k}(x)=\min _{u} \tilde{V}_{k+1}\left(f_{k}(x, u)\right)+L_{k}(x, u),
$$

and $\tilde{V}_{N}=V_{f}$. We call $\tilde{V}_{k}$ an unconstrained value function and distinguish it from the corresponding value function $V_{k}$. A solution to the unconstrained problem defines a feedback law $\kappa_{k}: \mathbb{R}^{n} \rightarrow \mathbb{R}^{m}$ satisfying,

$$
\kappa_{k}\left(\tilde{x}_{k}^{*}\right)=\tilde{u}_{k}^{*}
$$

where $\tilde{u}_{k}^{*}$ is a minimizer of (3a) and can be arbitrarily chosen. The unconstrained value function therefore satisfies,

$$
\tilde{V}_{k}(x)=\tilde{V}_{k+1}\left(f_{k}\left(x, \kappa_{k}(x)\right)\right)+L_{k}\left(x, \kappa_{k}(x)\right) .
$$

Consider the optimization problem,

$$
\begin{aligned}
\min _{\hat{u}} & \sum_{k=0}^{N-1} \Delta \tilde{V}_{k}\left(x_{k}, \hat{u}_{k}\right), \\
\text { sub. to } & x_{k+1}=\hat{f}_{k}\left(x_{k}, \hat{u}_{k}\right), \\
& \left(x_{k}, \kappa_{k}\left(x_{k}\right)+\hat{u}_{k}\right) \in \mathcal{C}_{k}, \forall k \in \mathbb{Z}_{N}, \\
& x_{N} \in \mathcal{X}_{N},
\end{aligned}
$$

where $x_{0}$ is given and,

$$
\Delta \tilde{V}_{k}\left(x_{k}, \hat{u}_{k}\right)=\tilde{V}_{k+1}\left(\hat{f}_{k}\left(x_{k}, \hat{u}_{k}\right)\right)+\hat{L}_{k}\left(x_{k}, \hat{u}_{k}\right)-\tilde{V}_{k}\left(x_{k}\right),
$$

with $\hat{f}_{k}(x, \hat{u})=f_{k}\left(x, \kappa_{k}\left(x_{k}\right)+\hat{u}\right)$ and $\hat{L}_{k}(x, \hat{u})=L_{k}\left(x, \kappa_{k}\left(x_{k}\right)+\hat{u}\right)$. Note that no element used in the construction of the objective function $(7)$ depends on constraints. We are now ready to state the main result.

Theorem 1 Let the sequence of control inputs $\hat{u}^{*}$ solve the optimization problem (6). Then the sequence of control inputs $u^{*}$ satisfying,

$$
u_{k}^{*}=\kappa_{k}\left(\hat{x}_{k}^{*}\right)+\hat{u}_{k}^{*}
$$

for all $k \in \mathbb{Z}_{N}$, solves the optimization problem (1), where $\hat{x}_{k+1}^{*}=\hat{f}_{k}\left(\hat{x}_{k}^{*}, \hat{u}_{k}^{*}\right)$ and $\hat{x}_{0}^{*}=x_{0}$.

PROOF. The sequence $\hat{u}^{*}$ satisfies,

$$
\hat{u}_{k}^{*} \in \underset{u \in \overline{\mathcal{C}}_{k}\left(\hat{x}_{k}^{*}\right)-\left\{\kappa_{k}\left(\hat{x}_{k}^{*}\right)\right\}}{\arg \min } \hat{V}_{k+1}\left(\hat{f}_{k}\left(\hat{x}_{k}^{*}, u\right)\right)+\Delta \tilde{V}_{k}\left(\hat{x}_{k}^{*}, u\right),
$$


where $\hat{V}_{k}$ satisfies the Bellman equation,

$$
\hat{V}_{k}(x)=\min _{u \in \overline{\mathcal{C}}_{k}(x)-\left\{\kappa_{k}(x)\right\}} \hat{V}_{k+1}\left(\hat{f}_{k}(x, u)\right)+\Delta \tilde{V}_{k}(x, u),
$$

with domain $\operatorname{Proj}_{\mathbb{R}^{n}}\left(\overline{\mathcal{C}}_{k}-\left\{\left(0, \kappa_{k}(x)\right)\right\}\right)=\operatorname{Proj}_{\mathbb{R}^{n}} \overline{\mathcal{C}}_{k}=\overline{\mathcal{X}}_{k}$, and $\hat{V}_{N}=\left.0\right|_{\mathcal{X}_{N}}$.

Let $\check{V}_{k}=\tilde{V}_{k}+\hat{V}_{k}, k \in \mathbb{Z}_{N+1}$. Then, according to (7),

$$
\tilde{V}_{k}(x)+\hat{V}_{k+1}\left(\hat{f}_{k}(x, u)\right)+\Delta \tilde{V}_{k}(x, u)=\check{V}_{k+1}\left(\hat{f}_{k}(x, u)\right)+\hat{L}_{k}(x, u) .
$$

According to (9b) and (10),

$$
\begin{aligned}
\check{V}_{k}(x) & =\min _{u \in \mathcal{C}_{k}(x)-\left\{\kappa_{k}(x)\right\}} \check{V}_{k+1}\left(\hat{f}_{k}(x, u)\right)+\hat{L}_{k}(x, u) \\
& =\min _{u \in \mathcal{C}_{k}(x)-\left\{\kappa_{k}(x)\right\}} \check{V}_{k+1}\left(f_{k}\left(x, \kappa_{k}(x)+u\right)\right)+L_{k}\left(x, \kappa_{k}(x)+u\right) .
\end{aligned}
$$

Fix $k \in \mathbb{Z}_{N}$ and assume $\check{V}_{k+1}=V_{k+1}$. Comparing (2b) to (11), we see that they are equivalent and therefore $\check{V}_{k}=V_{k}$. Since $\hat{V}_{N}=0$, then $\check{V}_{N}=V_{N}$. Therefore $\check{V}_{k}=V_{k}$ for all $k \in \mathbb{Z}_{N+1}$. According to (9a) and (10),

$$
\begin{aligned}
\hat{u}_{k}^{*} & \in \underset{u \in \overline{\mathcal{C}}_{k}\left(\hat{x}_{k}^{*}\right)-\left\{\kappa_{k}\left(\hat{x}_{k}^{*}\right)\right\}}{\arg \min } \check{V}_{k+1}\left(\hat{f}_{k}\left(\hat{x}_{k}^{*}, u\right)\right)+\hat{L}_{k}\left(\hat{x}_{k}^{*}, u\right) \\
& =-\kappa_{k}\left(\hat{x}_{k}^{*}\right)+\underset{u \in \overline{\mathcal{C}}_{k}\left(\hat{x}_{k}^{*}\right)}{\arg \min } V_{k+1}\left(f_{k}\left(\hat{x}_{k}^{*}, u\right)\right)+L_{k}\left(\hat{x}_{k}^{*}, u\right),
\end{aligned}
$$

in which the second expression was obtained by performing a change of variables $\kappa_{k}\left(\hat{x}_{k}^{*}\right)+u \mapsto u$.

Fix $k \in \mathbb{Z}_{N}$ and assume $\hat{x}_{k}^{*}=x_{k}^{*}$. Then the minimizer expressions in (2a) and (12) are equivalent, implying that there exists $u_{k}^{*}$ minimizing (2a) where $u_{k}^{*}=\kappa_{k}\left(\hat{x}_{k}^{*}\right)+\hat{u}_{k}^{*}$ and $\hat{x}_{k+1}^{*}=f_{k}\left(\hat{x}_{k}^{*}, \kappa_{k}\left(\hat{x}_{k}^{*}\right)+\hat{u}_{k}^{*}\right)=f_{k}\left(x_{k}^{*}, u_{k}^{*}\right)=x_{k+1}^{*}$. Since $\hat{x}_{0}^{*}=x_{0}=x_{0}^{*}$, we deduce that there exists a sequence $u^{*}$ solving (1) and satisfying $u_{k}^{*}=\kappa_{k}\left(\hat{x}_{k}^{*}\right)+\hat{u}_{k}^{*}$ for all $k \in \mathbb{Z}_{N}$.

\section{Linear MPC optimization problem}

In practical application, the result of Theorem 1 is most useful in instances where there exists an analytical solution to the unconstrained version of the optimization problem (1), or one in which the feedback law $\kappa_{k}\left(x_{k}\right)$ is conveniently parametrizable in terms of the state $x_{k}$. This is in particular true in the case of LQR and we consider this case in further detail by assuming a locally linear-quadratic structure to the problem (1),

$$
\begin{aligned}
f_{k}(x, u) & =A_{k} x+B_{k} u+o(\|(x, u)\|), \\
L_{k}(x, u) & =\frac{1}{2}\left[\begin{array}{ll}
x^{\mathrm{T}} & u^{\mathrm{T}}
\end{array}\right]\left[\begin{array}{ll}
Q_{k} & N_{k} \\
N_{k}^{\mathrm{T}} & R_{k}
\end{array}\right]\left[\begin{array}{l}
x \\
u
\end{array}\right]+o\left(\|(x, u)\|^{2}\right), \\
V_{f}(x) & =\frac{1}{2} x^{\mathrm{T}} P_{f} x+o\left(\|x\|^{2}\right) .
\end{aligned}
$$

We assume $P_{f}$ is positive definite and introduce,

$$
\begin{aligned}
K_{k} & =-\left(R_{k}+B_{k}^{\mathrm{T}} P_{k+1} B_{k}\right)^{-1}\left(B_{k}^{\mathrm{T}} P_{k+1} A_{k}+N_{k}^{\mathrm{T}}\right), \\
P_{k} & =A_{k}^{\mathrm{T}} P_{k+1} A_{k}+Q_{k}+\left(A_{k}^{\mathrm{T}} P_{k+1} B_{k}+N_{k}\right) K_{k},
\end{aligned}
$$

defined for $k \in \mathbb{Z}_{N}$, with $P_{N}=P_{f}$, and assume that $P_{k}$ and $R_{k}+B_{k}^{\mathrm{T}} P_{k+1} B_{k}$ are positive definite for all $k \in \mathbb{Z}_{N}$. 
Lemma 2 Consider the optimization problem (1) and assume that $f_{k}, L_{k}$, and $V_{f}$ are of the forms given in (13). Then,

$$
\Delta \tilde{V}_{k}(x, \hat{u})=\frac{1}{2} \hat{u}^{T}\left(R_{k}+B_{k}^{\mathrm{T}} P_{k+1} B_{k}\right) \hat{u}+o\left(\|(x, \hat{u})\|^{2}\right) .
$$

PROOF. Fix $k \in \mathbb{Z}_{N}$ and assume $\tilde{V}_{k+1}(x)=\frac{1}{2} x^{\mathrm{T}} P_{k+1} x+o\left(\|x\|^{2}\right)$. Then,

$$
\tilde{V}_{k}(x)=\min _{u} \frac{1}{2}(A x+B u)^{\mathrm{T}} P_{k+1}(A x+B u)+\frac{1}{2}\left[\begin{array}{ll}
x^{\mathrm{T}} & u^{\mathrm{T}}
\end{array}\right]\left[\begin{array}{ll}
Q_{k} & N_{k} \\
N_{k}^{\mathrm{T}} & R_{k}
\end{array}\right]\left[\begin{array}{l}
x \\
u
\end{array}\right]+o\left(\|(x, u)\|^{2}\right) .
$$

As shown by Dorato and Levis (1971), the unique minimizer of (16) is given by $u=K_{k} x+o(\|x\|)$ and,

$$
\tilde{V}_{k}(x)=\frac{1}{2} x^{\mathrm{T}} P_{k} x+o\left(\|x\|^{2}\right) .
$$

Since $V_{N}=V_{f}$, we can deduce that (17) is true for all $k \in \mathbb{Z}_{N}$. According to (7),

$$
\Delta \tilde{V}_{k}(x, \hat{u})=\frac{1}{2}\left[\begin{array}{ll}
x^{\mathrm{T}} & \hat{u}^{\mathrm{T}}
\end{array}\right]\left[\begin{array}{cc}
\hat{Q}_{k} & \hat{N}_{k} \\
\hat{N}_{k}^{\mathrm{T}} & \hat{R}_{k}
\end{array}\right]\left[\begin{array}{l}
x \\
\hat{u}
\end{array}\right]+o\left(\|(x, \hat{u})\|^{2}\right),
$$

where,

$$
\begin{aligned}
& \hat{Q}_{k}=\hat{A}_{k}^{\mathrm{T}} P_{k+1} \hat{A}_{k}+Q_{k}+K_{k}^{\mathrm{T}} N_{k}^{\mathrm{T}}+N_{k} K_{k}+K_{k}^{\mathrm{T}} R_{k} K_{k}-P_{k}, \\
& \hat{N}_{k}=\hat{A}_{k}^{\mathrm{T}} P_{k+1} B_{k}+N_{k}+K_{k}^{\mathrm{T}} R_{k}, \\
& \hat{R}_{k}=B_{k}^{\mathrm{T}} P_{k+1} B_{k}+R_{k},
\end{aligned}
$$

and $\hat{A}_{k}=A_{k}+B_{k} K_{k}$. Note that (14a) and (14b) imply that $\hat{N}_{k}=0$ and $\hat{Q}_{k}=0$, respectively.

Consider the following optimization problem,

$$
\begin{aligned}
\min _{\hat{u}} & \frac{1}{2} \sum_{k=0}^{N-1} \hat{u}_{k}^{\mathrm{T}}\left(R_{k}+B_{k}^{\mathrm{T}} P_{k+1} B_{k}\right) \hat{u}_{k}, \\
\text { sub. to } & x_{k+1}=\hat{A}_{k} x_{k}+B_{k} \hat{u}_{k}, \\
& \left(x_{k}, K_{k} x_{k}+\hat{u}_{k}\right) \in \mathcal{C}_{k}, \forall k \in \mathbb{Z}_{N}, \\
& x_{N} \in \mathcal{X}_{N},
\end{aligned}
$$

with $x_{0}$ given. The following result is a straightforward application of Theorem 1.

Corollary 3 Consider (1) and assume that $f_{k}, L_{k}$, and $V_{f}$ are of the forms given in (13) with no residual terms, i.e., $o=0$. Let the sequence of control inputs $\hat{u}^{\prime}$ solve (18). Then the sequence of control inputs $u^{\prime}$ satisfying,

$$
u_{k}^{\prime}=K_{k} \hat{x}_{k}^{\prime}+\hat{u}_{k}^{\prime}
$$

for all $k \in \mathbb{Z}_{N}$, solves the optimization problem $(1)$, where $\hat{x}_{k+1}^{\prime}=\hat{A}_{k} \hat{x}_{k}^{\prime}+B_{k} \hat{u}_{k}^{\prime}$ and $\hat{x}_{0}^{\prime}=x_{0}$.

\section{Constraint-separation principle}

We refer to the result of Corollary 3 as a constraint-separation principle, because it separates constraint enforcement from stabilization in MPC. The result implies that any locally linear-quadratic, open-loop MPC problem can be 
locally restructured as a closed-loop, constraint-enforcing, minimum-norm projection problem, where the feedback gain of the closed-loop controller is the optimal gain obtained by solving the unconstrained, open-loop MPC problem.

Importantly, this equivalence shows that linear MPC can be interpreted as an application of a constraint enforcement scheme to an already precompensated system, an interpretation that is associated with reference and command governors (Garone et al., 2017) and, in addition to being applied to reference commands, is what supposedly, significantly distinguishes these methods from MPC. It also shows that the extended command governor (ECG) (Gilbert and Ong, 2011), if applied as an offset to a control input similarly to the approach taken by Rossiter et al. (2010), can be viewed as a generalization of linear MPC, because the problem (18) is a special case of the conventional ECG optimization with reference input set to 0

The result also provides a method by which to choose the MPC penalty function when applying the decomposition technique to precompensated systems. For example, given a sequence of gains $K_{k}$, the penalty matrix should be set to $R_{k}+B_{k}^{\mathrm{T}} P_{k+1} B_{k}$, where $R_{k}$ and $P_{k}$ satisfy the solution of an inverse LQR problem obtained, for example, using methods of Kreindler and Jameson (1972); Di Cairano and Bemporad (2010). In the nonlinear case, a solution to a general, inverse optimal control problem could be used to determine a cost function for an MPC controller with the cost function $\Delta V_{k}$ being determined according to the results of the main theorem.

Additionally, as explored by Kouvaritakis et al. (2000, 2002); Rossiter (2004), the result provides a potential simplification to numerical approaches to MPC problems. As discussed by Diehl et al. (2009), most conventional numerical approaches to nonlinear MPC problems either apply a sequential quadratic programming approach, where the corollary is obviously useful, or an interior point approach, where the main theorem is more useful since precompensation at least allows for better numerical conditioning as it prevents the closed-loop maps $\hat{f}_{k}$ from blowing up. To the best of our knowledge, most solvers applied to MPC, although allowing for the option to precompensate according to the optimal feedback, e.g., Kvasnica et al. (2006), do not fully take advantage of the separation principle presented. For example, they do not decompose the local problem into what it essentially consists of: an LQR problem and a minimum-norm projection onto a closed set. They instead allow for a user to precompensate according to any feedback when the choice of optimal feedback has greater utility, as it guarantees no error when constraints are inactive.

The discussion thus far has focused on the special case where the MPC problem is locally linear-quadratic. We note that the main result is applicable more generally, as represented by the schematic in Fig. 2, which we have shown to be equivalent to Fig. 1. The constraint-separation principle does not necessarily hold in the general case, when the problem is not locally linear-quadratic, e.g., when there does not exist a continuously differentiable stabilizing feedback $\kappa_{k}$ or the cost function $V_{k}$ is not continuously differentiable; in this case, it is not guaranteed that the penalty function $\Delta \tilde{V}_{k}$ is locally independent of $x$. Nevertheless, the generality of the main result is remarkable: The result can be applied as long as a solution is known to the unconstrained problem, with a few minor, technical conditions corresponding to constraints and penalty function. However, note that constraint-enforcement does not necessarily simplify to a minimum-norm projection in this case.

Now note the practical use of decomposition in certifying MPC controllers. A decomposed controller is more straightforward to certify because an unconstrained problem is simpler than the same problem with constraints; it is therefore easier to certify the stabilizing component of the decomposed MPC problem. This makes it easier to ensure stability and attractiveness during online operation, limiting the need for a complex certification process, such as that of (Davy et al., 2018), to the constraint-enforcement component.

Taken together, the above discussion implies that there is a strong desire to often, if not always, decompose design in the manner derived in this manuscript, whenever one is able to effectively parametrize the feedback controller $\kappa_{k}$ and the closed-loop state update $\hat{f}_{k}$. This approach can, for example, improve design of neural-net-based MPC (Saint-Donat et al., 1991) by decomposing the problem into the solution of a simpler, unconstrained, approximate dynamic programming problem (Bertsekas, 2014), and a more difficult, constrained optimization problem.

As a matter of course, this discussion has only been able to superficially consider the practical use of decomposition in nonlinear MPC. We feel, however, that it represents a promising direction for future research.

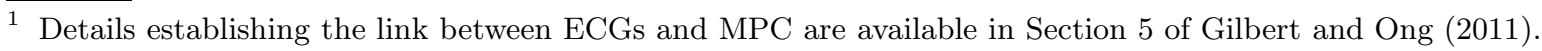




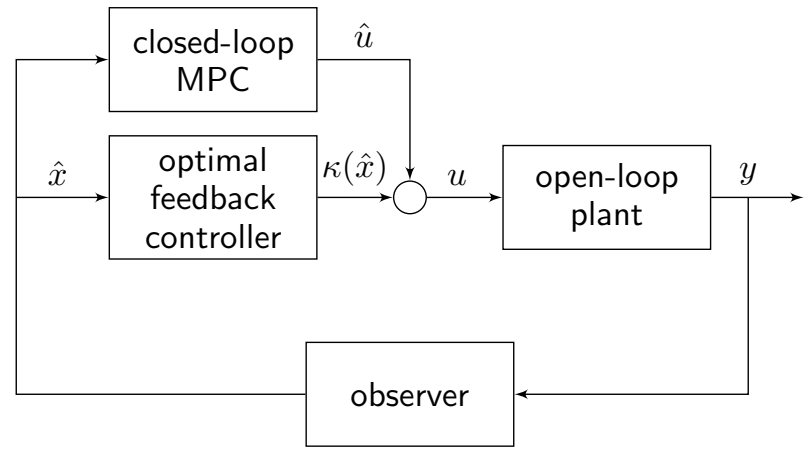

Fig. 2. MPC decomposed into an optimal feedback controller and a constraint-enforcing scheme

\section{Conclusion}

In this brief, we derived a constraint-separation principle for MPC problems. The results show that MPC problems can be decomposed into the solution of an unconstrained, open-loop problem and a constrained, closed-loop problem without a terminal cost, which may simplify MPC problems when the unconstrained, stabilizing feedback can be represented explicitly by a closed-form solution or a parametrization. This is particularly true for linear MPC problems, for which the stabilizing feedback is given as the solution to the well-known LQR problem. It is significant because it shows the equivalence of designing MPC in a two-step approach, which first stabilizes the system and then implements constraint protection in the outer-loop. It is also significant because it can be used to improve numerical solutions to both linear and nonlinear MPC problems.

\section{References}

D. P. Bertsekas. Dynamic Programming and Optimal Control, volume 2. Athena Scientific, Nashua, NH, 4th edition, 2014.

G. Davy, E. Feron, P.-L. Garoche, and D. Henrion. Experiments in verification of linear model predictive control: Automatic generation and formal verification of an interior point method algorithm. In Proc. Int. Conf. Logic for Programming, Artificial Intelligence and Reasoning, pages 290-306, Awassa, Ethiopia, 2018.

S. Di Cairano and A. Bemporad. Model predictive control tuning by controller matching. IEEE Trans. Automat. Contr., 55(1):185-190, 2010.

M. Diehl, H. J. Ferreau, and N. Haverbeke. Efficient numerical methods for nonlinear MPC and moving horizon estimation. In L. Magni, D. M. Raimondo, and F. Allgöwer, editors, Nonlinear Model Predictive Control: Towards New Challenging Applications, pages 391-417. Springer-Verlag, Berlin, 2009.

P. Dorato and A. H. Levis. Optimal linear regulators: The discrete-time case. IEEE Trans. Automat. Contr., 16(6): 613-620, 1971.

E. Garone, S. Di Cairano, and I. Kolmanovsky. Reference and command governors for systems with constraints: A survey on theory and applications. Automatica, 75:306-328, 2017.

E. G. Gilbert and C.-J. Ong. Constrained linear systems with hard constraints and disturbances: An extended command governor with large domain of attraction. Automatica, 47(2):334-340, 2011.

M. V. Kothare, P. J. Campo, M. Morari, and C. N. Nett. A unified framework for the study of anti-windup designs. Automatica, 30(12):1869-1883, 1994.

B. Kouvaritakis, J. A. Rossiter, and J. Schuurmans. Efficient robust predictive control. IEEE Trans. Automat. Contr., 45(8):1545-1549, 2000.

B. Kouvaritakis, M. Cannon, and J. A. Rossiter. Who needs QP for linear MPC anyway? Automatica, 38(5):879-884, 2002.

E. Kreindler and A. Jameson. Optimality of linear control systems. IEEE Trans. Automat. Contr., 17(3):349-351, 1972.

M. Kvasnica, P. Grieder, M. Baotić, and F. J. Christophersen. Multi-Parametric Toolbox (MPT). Automatic Control Laboratory, Swiss Federal Institute of Technology, Zurich, 2006.

J. B. Rawlings, D. Q. Mayne, and M. M. Diehl. Model Predictive Control: Theory, Computation, and Design. Nob Hill Publishing, Santa Barbara, CA, 2nd edition, 2017.

J. A. Rossiter. Model-Based Predictive Control: A Practical Approach. CRC Press, Boca Raton, FL, 2004.

J. A. Rossiter, L. Wang, and G. Valenica-Palomo. Efficient algorithms for trading off feasibility and performance in predictive control. Int. J. Control, 84(4):789-797, 2010. 
J. Saint-Donat, N. Bhat, and T. J. McAvoy. Neural net based model predictive control. Int. J. Control, 54(6): 1453-1468, 1991.

K. P. Tee, S. S. Ge, and E. H. Tay. Barrier Lyapunov functions for the control of output constrained nonlinear systems. Automatica, 45(4):918-927, 2009. 